\title{
DN Tauri - coronal activity and accretion in a young low-mass CTTS
}

\author{
J. Robrade ${ }^{1}$, M. Güdel ${ }^{2}$, H. M. Günther ${ }^{3}$, and J. H. M. M. Schmitt ${ }^{1}$ \\ 1 Hamburger Sternwarte, University of Hamburg, Gojenbergsweg 112, 21029 Hamburg, Germany \\ 2 Department of Astronomy, University of Vienna, Türkenschanzstr. 17, 1180 Vienna, Austria \\ 3 Harvard-Smithsonian Center for Astrophysics, 60 Garden Street, Cambridge, MA 02138, USA \\ e-mail: jrobrade@hs.uni-hamburg.de
}

Received 24 September 2013 / Accepted 14 November 2013

\section{ABSTRACT}

\begin{abstract}
Context. Classical T Tauri stars (CTTSs) are young, accreting low-mass stars; their X-ray emission differs from that of their mainsequence counterparts in a number of aspects.

Aims. We study the specific case of DN Tau, a young M0-type accreting CTTS, to extend the range of young CTTSs studied with high-resolution X-ray spectroscopy at lower masses and to compare its high-energy properties with those of similar objects.

Methods. We use a deep XMM-Newton observation of DN Tau to investigate its X-ray properties and X-ray generating mechanisms. Specifically, we examine the presence of X-ray emission from magnetic activity and accretion shocks. We also compare our new X-ray data with UV data taken simultaneously and with X-ray/UV observations performed before.

Results. We find that the X-ray emission from DN Tau is dominated by coronal plasma generated via magnetic activity, but also clearly detect a contribution of the accretion shocks to the cool plasma component at $\lesssim 2 \mathrm{MK}$ as consistently inferred from density and temperature analysis. Typical phenomena of active coronae, such as flaring, the presence of very hot plasma at $30 \mathrm{MK}$, and an abundance pattern showing the inverse FIP effect, are seen on DN Tau. Strong variations in the emission measure of the cooler plasma components between the 2005 and 2010 data point to accretion related changes; in contrast, the hotter coronal plasma component is virtually unchanged. The UV light curve taken simultaneously is in general not related to the X-ray brightness, but exhibits clear counterparts during the observed X-ray flares.

Conclusions. The X-ray properties of DN Tau are similar to those of more massive CTTSs, but its low mass and large radius associated with its youth shift the accretion shocks to lower temperatures, reducing their imprint in the X-ray regime. DN Tau's overall X-ray properties are dominated by strong magnetic activity.
\end{abstract}

Key words. stars: individual: DN Tauri - stars: pre-main sequence - stars: activity - stars: coronae - X-rays: stars

\section{Introduction}

Classical T Tauri stars (CTTSs) are pre-main sequence, lowmass stars $\left(M \lesssim 2 M_{\odot}\right)$ that are still accreting matter from a surrounding circumstellar disk at a significant level. CTTSs show an emission line spectrum and are often characterized by strong veiling and a large $\mathrm{H} \alpha$ equivalent width, formerly the main classifier of this class. The $\mathrm{H} \alpha$ equivalent width criterion for CTTSs is, however, spectral-type dependent. Further studies have shown that the $10 \%$ width of the $\mathrm{H} \alpha$ line is mostly determined by the accretion streams and is less spectral-type dependent and a more reliable tracer of accretion, especially in lowmass stars (see, e.g., White \& Basri 2003). Besides H $\alpha$, many other lines have been used to quantitatively study accretion properties in young stars. In addition to emission lines, CTTSs show a strong IR-excess from their circumstellar disk that also provides a diagnostic of the inclination of the system. CTTSs evolve via transitional objects, where the disk starts to dissipate and accretion rates decrease, into weak-line T Tauri stars (WTTSs) that are virtually disk-less and do not show strong signs of ongoing accretion.

T Tauri stars are known to be strong and variable X-ray emitters from Einstein and ROSAT observations. X-ray studies of star-forming regions, such as COUP (Chandra Orion Ultradeep Project, Getman et al. 2005) or XEST (XMM-Newton Extended Survey of the Taurus Molecular Cloud, Güdel et al. 2007a), confirmed that $\mathrm{T}$ Tauri stars show high levels of magnetic activity as shown by hot coronal plasma and strong flaring, but also refined the general X-ray picture of young stellar objects (YSOs).

In the commonly accepted magnetospheric accretion model for CTTSs (e.g., Koenigl 1991) material is accreted from the stellar disk onto the star along magnetic field lines, which disrupt the accretion disk in the vicinity of the corotation radius. Since the infalling material originates from the disc truncation radius, typically located at several stellar radii, it reaches almost free-fall velocity, and upon impact the supersonic flow forms a strong shock near the stellar surface. The funnelling of the accreted matter by the magnetic field leads to the formation of accretion spots that have small surface filling factors (Calvet \& Gullbring 1998) and produce strong optical/UV and X-ray emission (Lamzin 1998; Günther et al. 2007).

The X-ray emission from accretion spots on CTTSs has specific signatures that are detectable with high-resolution X-ray spectroscopy. Accretion shocks generate plasma with temperatures of up to a few MK that is significantly cooler than the average coronal plasma and sufficiently funneled accretion streams are expected to produce X-rays in a high-density environment ( $n_{\mathrm{e}} \gtrsim 10^{11} \mathrm{~cm}^{-3}$ measured in O VII). In contrast, plasma produced by magnetic activity covers a much broader temperature range spanning in total $1-100 \mathrm{MK}$ and is on average hotter ( $T_{\mathrm{av}} \approx 10-20 \mathrm{MK}$ for CTTSs in Taurus, Telleschi et al. 2007). Furthermore, it typically has, at least for outside large flares, much lower densities $\left(n_{\mathrm{e}} \lesssim 3 \times 10^{10} \mathrm{~cm}^{-3}\right.$, Ness et al. 2004). 
The accretion streams may also influence coronal structures on the stellar surface or lead to additional magnetic activity via stardisk interaction. Moreover, the accretion process is accompanied by outflows or winds from the star and the surrounding disk, which play an important role in star formation via the transport of angular momentum. Stellar jets and associated shocks provide another X-ray production mechanism, which typically generates cool plasma at low densities, as seen in several T Tauri stars such as DG Tau (Güdel et al. 2005). X-ray diagnostics, such as density and temperature-sensitive $\mathrm{X}$-ray line ratios, can be utilized to distinguish between the different scenarios.

TW Hya was the first and is still the most prominent CTTS that is dominated by accretion shocks in X-rays. Its X-ray spectrum shows high-density plasma as shown by density-sensitive lines in He-like triplets of oxygen and neon and an unusually cool plasma distribution (Kastner et al. 2002; Stelzer \& Schmitt 2004). TW Hya has been extensively studied in X-rays, and a very deep Chandra observation suggests that the accreted and shock-heated material mixes with surrounding coronal material, very likely producing a complex distribution of emission regions around the accretion spots (Brickhouse et al. 2010). So far all low-mass CTTSs studied at X-ray energies have shown similar signs of accretion plasma. The classic examples are, for example, BP Tau (Schmitt et al. 2005), V4046 Sgr (Günther et al. 2006; Argiroffi et al. 2012), MP Mus (Argiroffi et al. 2007) or RU Lup (Robrade \& Schmitt 2007). In contrast, T Tau itself shows a strong cool plasma component, but low plasma density (Güdel et al. 2007b); although the system is dominated by the intermediate-mass $\mathrm{T}$ Tauri star $\mathrm{T}$ Tau $\mathrm{N}\left(M \approx 2.4 M_{\odot}\right)$. In a comparative study of several bright CTTSs, it was shown that the presence of X-rays from both accretion shocks and magnetic activity is most likely universal, but that the respective contributions differ significantly between the individual objects (Robrade \& Schmitt 2006). Indeed, magnetic activity produces the bulk of the observed X-ray emission in the majority of CTTSs in the $0.2-2.0 \mathrm{keV}$ band and completely dominates at higher energies. In addition, X-ray temperature diagnostics have shown that all accreting stars exhibit an excess of shockgenerated cooler plasma, leading to a soft excess when compared to coronal sources (Robrade \& Schmitt 2007; Telleschi et al. 2007; Güdel \& Telleschi 2007). The observed X-ray spectrum of young stars is modified by often significant absorption by circumstellar or disk material, as well as by outflowing and infalling matter. X-ray absorption can exceed optical extinction by an order of magnitude, as shown, say, for RU Lup (Robrade \& Schmitt 2007) and may even be strongly time variable as in AA Tau (Schmitt \& Robrade 2007). The different stellar properties (such as mass, rotation, and activity), varying mass accretion rates, and degree of funneling, as well as the viewing angle dependence, naturally lead to the variety of X-ray phenomena in YSOs that are an intermixture of magnetic activity, accretion, and outflow processes.

High-resolution X-ray spectra from young accreting stars are available only in a few cases, and existing studies focused on the more massive CTTSs with spectral type G or K. Young lowmass stars with $M \lesssim 0.5 M_{\odot}$ are typically X-ray fainter and have so far only been poorly studied; the transitional multiple system Hen 3-600 (Huenemoerder et al. 2007) is one of the rare exceptions. Nevertheless, they are the most common stars, and their investigation is of great astrophysical interest to draw a more complete and general picture of the evolution of young stars and their surrounding environment, where the stellar winds and $\mathrm{UV} / \mathrm{X}$-ray emission influence the chemistry and evolution of the circumstellar disk and the process of planet formation.
Table 1. Stellar properties of DN Tau from optical measurements.

\begin{tabular}{lcc}
\hline \hline Sp. type & $\mathrm{M} 0^{1,2,3}$ & \\
$T_{\text {eff }}$ & $3800^{3} \ldots 3850^{1} \ldots 3950 \pm 50^{4}$ & $\mathrm{~K}$ \\
$M_{*}$ & $0.4^{1,2} \ldots 0.5^{3} \ldots 0.65 \pm 0.05^{4}$ & $M_{\odot}$ \\
$R_{*}$ & $1.9 \pm 0.2^{4} \ldots 2.1^{2}$ & $R_{\odot}$ \\
$L_{\mathrm{bol}}$ & $0.8 \pm 0.2^{4} \ldots 0.9^{2} \ldots 1.0^{1}$ & $L_{\odot}$ \\
$A_{V}$ & $0.25^{2} \ldots 0.5^{1}$ & $\mathrm{mag}$ \\
$\log \dot{M}_{\text {acc }}$ & $-7.8^{3} \ldots-8.5^{2} \ldots-9.1 \pm 0.3^{4}$ & $M_{\odot} \mathrm{yr}^{-1}$ \\
\hline
\end{tabular}

References. ${ }^{(1)}$ Kenyon \& Hartmann (1995); (2) Gullbring et al. (1998); (3) White \& Hillenbrand (2004); ${ }^{(4)}$ Donati et al. (2013).

\section{The target: DN Tau}

Our target star DN Tau is an M0-type CTTS located in the Taurus Molecular Cloud (TMC) at a distance of $d=140 \mathrm{pc}$ (Cohen \& Kuhi 1979); important stellar parameters collected from the literature are summarized in Table 1. DN Tau is a single star on a fully convective track with an estimated age in the range of 0.5-1.7 Myr. Its optical extinction is quite low, indicating that DN Tau is not deeply embedded in circumstellar material or the TMC. While classical estimates of stellar luminosity, mass, and radius are about $L_{*}=1.0 L_{\odot}, M_{*}=0.5 M_{\odot}$, and $R_{*}=2.1 R_{\odot}$, Donati et al. (2013) find a slightly hotter, smaller, and less luminous model of DN Tau $\left(L_{*}=0.8 L_{\odot}\right.$, $M_{*}=0.65 M_{\odot}, R_{*}=1.9 R_{\odot}$ ) by adopting an optically measured $A_{V}=0.5$. In contrast, Ingleby et al. (2013) find a much brighter and larger DN Tau $\left(L_{*}=1.5 L_{\odot}, M_{*}=0.6 M_{\odot}, R_{*}=2.8 R_{\odot}\right)$ when using $A_{V}=0.9$, that are based on $A_{J}=0.29$ from IRmeasurements (Furlan et al. 2011). The CTTS nature of DN Tau is reflected by a typical $E W[\mathrm{H} \alpha]=12-18 \AA$ and an $\mathrm{H} \alpha 10 \%$ width in the range of $290-340 \mathrm{~km} \mathrm{~s}^{-1}$ (Herbig \& Bell 1988; White \& Hillenbrand 2004; Nguyen et al. 2009) and a moderate IR excess, making it a Class II source based on its far-IR SED (Kenyon \& Hartmann 1995). DN Tau is a variable, but typically moderate accretor that exhibits little UV excess; e.g., Gullbring et al. (1998) derived $L_{\text {acc }}=0.016 L_{\odot}$ and a weak optical veiling of $r=0.075$. Nevertheless, the infalling plasma on DN Tau is apparently funnelled well with an accretion-spot filling factor of $f=0.005$ (Calvet \& Gullbring 1998). Ingleby et al. (2013) modeled broad-band optical and UV data in a similar approach but with multiple accretion columns and found, depending on the absence/presence of "hidden" low-flux accretion emission, $f=0.002 / 0.06$ and $\log \dot{M}_{\text {acc }}=-8 /-7.8 M_{\odot} \mathrm{yr}^{-1}$. Donati et al. (2013) give $\log \dot{M}_{\mathrm{acc}}=-9.1 \pm 0.3 M_{\odot} \mathrm{yr}^{-1}$ as the average for their accretion proxies. While the reliability of the various methods used to obtain quantitative estimates on the mass accretion rates is debated, highly variable accretion properties of DN Tau are observed and Fernandez et al. (1995) measured an EW [H $\alpha$ ] declining from 87 to $15 \AA$ within four days. DN Tau only has a weak outflow; while typically about $5-10 \%$ of the accretion rate are estimated, White $\&$ Hillenbrand (2004) give a $2 \%$ upper limit derived from their data.

Photometric variations in DN Tau's optical brightness were first reported with a period of about $P_{\text {rot }} \approx 6.0 \mathrm{~d}$ (Bouvier et al. 1986), and later refined to $P_{\text {rot }}=6.3 \mathrm{~d}$ (Vrba et al. 1993). This variability can be interpreted as rotational modulation of a large magnetic spot or spot group with a surface coverage of up to $35 \%$. Strong magnetic activity on DN Tau is also implied by its large inferred mean magnetic field of $2 \mathrm{kG}$ (Johns-Krull 2007). Results from spectropolarimetric observations with ESPaDOnS/CFHT (Donati et al. 2013) show a simple magnetic topology that is largely axisymmetric and mostly 
poloidal with a dominant octupolar and a weaker bipolar component of $0.6-0.8$. $\mathrm{kG}$ and $0.3-0.5 \mathrm{kG}$ polar strength. Muzerolle et al. (2003) present near-IR spectra of DN Tau from which they inferred an inner (dust-)disk rim located at $0.07 \mathrm{AU}\left(\approx 7 R_{*}\right)$, notably the closest disk rim in their sample. The disk of DN Tau with $M_{\mathrm{d}}=0.03 M_{\odot}$, as deduced from submillimeter observations, is quite massive, roughly an order of magnitude above the median mass found for the Class II sources in the sample of Andrews \& Williams (2005). DN Tau is viewed under an intermediate inclination; Muzerolle et al. (2003) inferred an inclination of $i=28 \pm 10^{\circ}$ from IR data, quite similar to the estimate of $i=35 \pm 10^{\circ}$ by Donati et al. (2013). Adopting $P_{\text {rot }}=6.3 \mathrm{~d}, i=33^{\circ}$ and combining these data with the rotational velocity of $v \sin i=12.3 \pm 0.6 \mathrm{~km} \mathrm{~s}^{-1}$ (Nguyen et al. 2009), $v \sin i=9 \pm 1 \mathrm{~km} \mathrm{~s}^{-1}$ (Donati et al. 2013) or $v \sin i=$ $10.2 \mathrm{~km} \mathrm{~s}^{-1}$ Hartmann \& Stauffer (1989), we obtain $R_{*} \approx 2.8 R_{\odot}$, $R_{*} \approx 2.0 R_{\odot}$, and $R_{*} \approx 2.3 R_{\odot}$, respectively.

X-ray emission from DN Tau was first detected with Einstein (Walter \& Kuhi 1981) and later by ROSAT (Neuhäuser et al. 1995), both at a similar X-ray luminosity of $\log L_{X} \approx$ $29.7 \mathrm{erg} \mathrm{s}^{-1}$, albeit with significant error. DN Tau has been observed by XMM-Newton in 2005 as part of the XEST project (No. 12-040); an analysis of these data is presented in Telleschi et al. (2007). They derived basic X-ray properties from an EMD model and a multitemperature fit, and both methods give $L_{\mathrm{X}}=1.2 \times 10^{30} \mathrm{erg} \mathrm{s}^{-1}$ and an average coronal temperature of about $12-14$ MK. DN Tau is among the X-ray brighter CTTSs, when compared to similar objects in the XEST or COUP sample, and its X-ray activity level is with $\log L_{\mathrm{X}} / L_{\mathrm{bol}} \approx-3.5$ close to, but still about a factor of three below, the saturation limit at $\log L_{\mathrm{X}} / L_{\mathrm{bol}} \approx-3$. We observed DN Tau again in 2010 with XMM-Newton, primarily to obtain a deeper exposed highresolution X-ray spectrum with the aim of expanding the sample of emission line studied CTTSs into the lower mass regime.

In this paper we present an analysis of the new XMM-Newton observations of DN Tau and compare it to earlier observations. Our paper is structured as follows. In Sect. 3 the X-ray observations and the data analysis are described, in Sect. 4 we present our results subdivided into different physical topics, in Sect. 5 we discuss our DN Tau results and compare it to other CTTSs and end with a summary in Sect. 6.

\section{Observations and data analysis}

The target DN Tau was observed by XMM-Newton twice. A $30 \mathrm{ks}$ exposure was obtained for the XEST survey in 2005 (PI: Guedel), and a $120 \mathrm{ks}$ exposure was obtained in 2010 (PI: Robrade). We focused on the deeper 2010 exposure, but also analyzed the 2005 data again in an identical fashion to ensure consistency throughout this work. Data were taken with all X-ray detectors, i.e., the EPIC (European Photon Imaging Camera) and the RGS (Reflection Grating Spectrometer), as well as the optical monitor (OM). The EPIC consists of two MOS and one $\mathrm{PN}$ detector. The PN is the more sensitive instrument, whereas the MOS detectors have a slightly higher spectral resolution. The EPIC instruments were operated in both observations in the full frame mode with the medium filter, allowing a direct comparison of the data. The OM was operated in fast mode with the $U$ filter in 2005 (eff. wavelength $3440 \AA$ ) and the $U V W 1$ (eff. wavelength $2910 \AA$ ) filter in 2010 . A detailed description of the instruments can be found in the "XMM-Newton Users Handbook"1; the used data is summarized in Table 2.

\footnotetext{
1 http://xmm.esac.esa.int
}

Table 2. XMM-Newton observing log of DN Tau.

\begin{tabular}{lcc}
\hline \hline Date & Obs. ID. & Dur. MOS/PN (ks) \\
\hline $2005-03-04 / 05$ & 0203542101 & $31 / 29$ \\
$2010-08-18 / 19$ & 0651120101 & $119 / 118$ \\
\hline
\end{tabular}

All data analysis was carried out with the XMM-Newton Science Analysis System (SAS) version $11.0^{2}$ and standard SAS tools were used to produce images, light curves, and spectra. Standard selection criteria were applied to the data, light curves are background subtracted, and we excluded periods of high background from spectral analysis. Source photons from the EPIC detectors were extracted from circular regions around DN Tau, and the background was taken from nearby source-free regions. The RGS data of DN Tau only has a moderate signalto-noise ratio $(\mathrm{S} / \mathrm{N})$, therefore we extracted spectra from a $90 \%$ PSF source region to reduce the background contribution. The data of the X-ray detectors were analyzed independently for each observation to study variability and cross-check the results from the different instruments. We note that some degradation has occurred for the RGS detector between the exposures, while the effective area of the EPIC detectors only shows minor changes.

Spectral analysis was carried out with XSPEC V12.6 (Arnaud 1996) and we used multi-temperature APEC/VAPEC models (Smith et al. 2001) with abundances relative to solar photospheric values as given by Grevesse \& Sauval (1998) to derive $\mathrm{X}$-ray properties like luminosities or emission measure distributions (EMD). We find that photoelectrically absorbed threetemperature models adequately describe the data, but note that some of the fit parameters are mutually dependent, such as absolute abundances and emission measure, emission measures and temperatures of neighboring components or absorption column density, temperature and emission measure of cool spectral components. Spectra were rebinned for modeling, and errors in spectral models are given by their $90 \%$ confidence range and were calculated by allowing variations of normalizations and respective model parameters. Additional uncertainties may arise from errors in the atomic data and instrumental calibration. For line fitting purposes we use the CORA program (Ness \& Wichmann 2002), identical line widths, and Lorentzian line shapes. Emitted line fluxes were corrected for absorption by using the ismtautool of the PINTofALE software (Kashyap \& Drake 2000) and flux-conversion was done with the SAS tool rgsfluxer.

\section{Results}

Here we report on the results obtained from the XMM-Newton observations, subdivided into separate topics.

\subsection{X-ray light curves and hardness}

The X-ray light curves of DN Tau as obtained from the summed EPIC data are shown in Fig. 1. Here we use the 0.2-5.0 keV energy band and a $1 \mathrm{ks}$ temporal binning. Some variability and minor activity is present in both observations, but large portions of the X-ray light curves are quite flat, and only one moderate flare visible at 55-60 ks with a factor two increase in count rate and two smaller events peaking at about $75 \mathrm{ks}$ and $95 \mathrm{ks}$ are detected during the 2010 exposure. The features at the beginning and end of the 2005 observation are also likely to be decay and rise phases of partly covered flares. Except for a higher average count

2 de la Calle, I. 2012, http: //xmm. esac.esa.int 


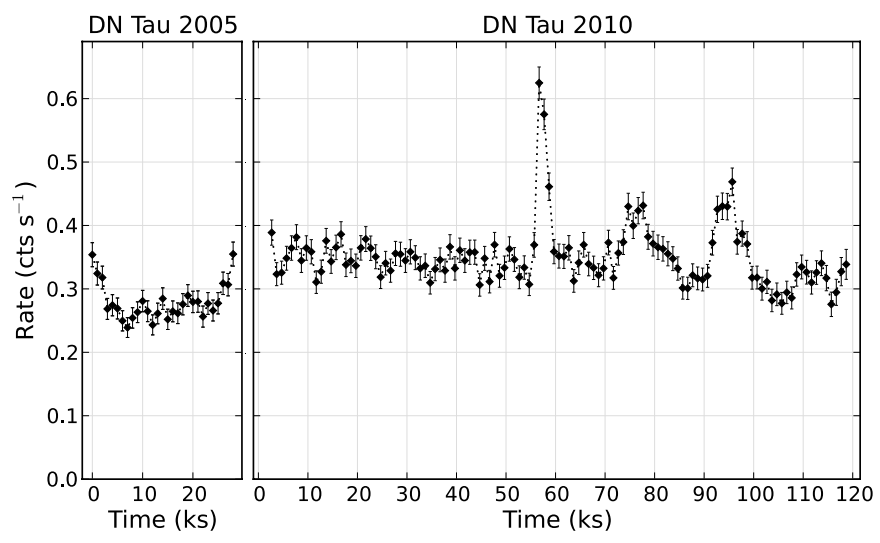

Fig. 1. X-ray light curves of DN Tau in 2005 and 2010, 0.2-5.0 keV EPIC data with 1 ks binning.

rate of $20 \%$ to $30 \%$ in 2010 , the level of variability within each observation period is comparable. A long-term trend toward declining X-ray count rate by roughly $10 \%$ is seen in the 2010 data and might be due to rotational modulation. The $1.4 \mathrm{~d}$ observation has a phase coverage of about 0.22 and, given the moderate inclination of DN Tau rotational modulation, can be expected for surface features at low and intermediate latitudes.

We investigated the basic spectral state of DN Tau for both exposures and its evolution with a hardness ratio analysis, $H R=$ $(H-S) /(H+S)$ with $0.2-0.8 \mathrm{keV}$ as soft band and $0.8-5.0 \mathrm{keV}$ as hard band. The energy bands were chosen in such a way that $\mathrm{X}$-ray emission in our soft band is predominantly produced by plasma at temperatures of 2-5 MK, whereas the hard band is dominated by emission from hotter plasma at 5-20 MK; however, a moderate shift of the band-separation energy does not influence the results. As shown in Fig. 3 the positive correlation between X-ray brightness and spectral hardness that is typically observed for magnetic activity is generally not present in DN Tau. We detect a spectral hardening during the larger flares in 2010, but overall a clear correlation between brightness and hardness is not present. Typically more active coronal stars exhibit harder spectra (see e.g. Schmitt 1997), but a similar trend is also seen when studying the temporal behavior in individual objects including CTTSs (Robrade \& Schmitt 2006). Remarkably, we find that the X-ray fainter state in the year 2005 is overall characterized by harder emission than the brighter 2010 state. In addition, the individual observation periods again show a broad scatter and only marginal correlations. Similar conclusions are obtained when inspecting the time evolution of the hardness ratio.

\subsection{UV light curves, flares and UV/X-ray correlations}

The OM light curves of DN Tau are plotted in Fig. 2, and the associated brightness errors are in the range of 0.01 mag and below the size of the shown symbols. The DN Tau observation from 2005 was performed in the $U$ band filter (NUV), while in 2010 the bluer, but less sensitive $U V W 1$ filter (NUV-MUV) was used.

If comparing the UV light curves with the X-ray ones (Fig. 1), it is evident that the short term variations of the 2005 $U$ band data do not strictly correlate with those of the X-ray brightness, suggesting a different origin of the respective emission. Similarly, Vrba et al. (1993) find the $U$ band brightness variations to be rather stochastic and not as modulated by the $6.3 \mathrm{~d}$ rotation period as the other optical bands $(B V R I)$.

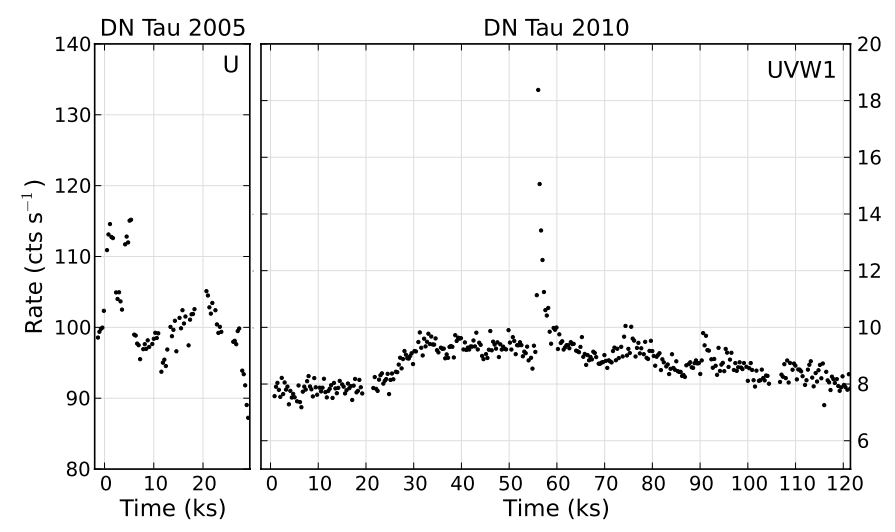

Fig. 2. OM light curves from 2005 ( $U$ filter) and 2010 (UVW1 filter), 300 s binning each.

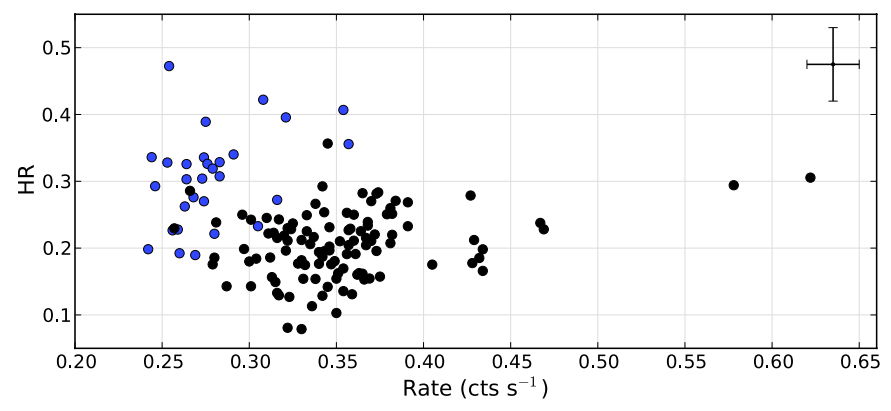

Fig. 3. Hardness ratio of DN Tau from EPIC data, 2010 (black), 2005 (blue); typical errors are indicated in the upper right corner.

This indicates that a significant fraction of the UV flux is not associated with the dark spots that are interpreted as magnetically active regions. Furthermore, except for a shorter observing period where an out-of-phase modulation was found, a stable accretion configuration, i.e. a dominant hot spot, was not present, and overall their $U$ band variations are much larger with \pm 0.6 mag than those in $B V R I$ with $0.1-0.2$ mag. Looking at long-term variations, DN Tau apparently brightened in the UV-range over the past decades without comparable changes in optical bands. While there is also significant variability on shorter timescales, the $U$ band brightness increased on average from about 14.5 (14.0-14.9) mag during the monitoring in the 1980s over 13.9 (14.3-13.3) mag in the early 1990s (Grankin et al. 2007) to a magnitude of 13.25 (13.12-13.36) mag (1.5 ks XMM-Newton average) in 2005.

Also the moderately "harder" $U V W 1$ flux during the quasiquiescent part of the 2010 observation is apparently not correlated with X-ray brightness. During our observation the $U V$ flux varies significantly on timescales of minutes to hours; for example, we observe at about $25 \mathrm{ks}$ an increase in the $U V W 1$ rate by roughly $20 \%$ within a few ks, but without any corresponding X-ray signature as might be expected for magnetic activity. Since the photospheric UV emission is negligible in M type stars, the observed behavior favors a scenario where the bulk of the UV emission is related to several accretion spots located on the surface of DN Tau, and variability is created by changes in geometry and/or variable spot brightness.

A rough estimate of the relative contributions from magnetic activity and accretion to the UV flux of DN Tau can be obtained by a comparison to purely magnetically active sources under the assumption of similar X-ray generating coronae and magnetically induced chromospheric UV emission. Here we use the active mid-M dwarf EV Lac (Mitra-Kraev et al. 2005) that was 


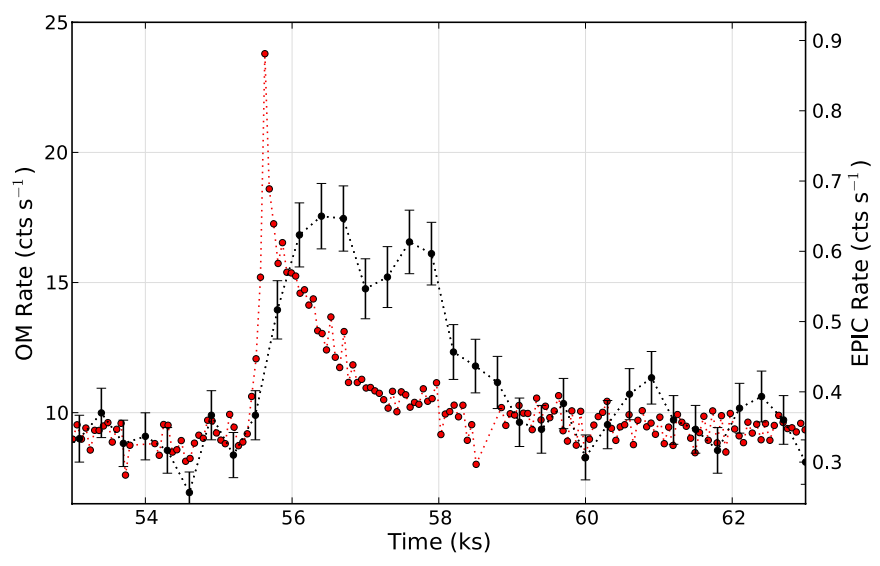

Fig. 4. The largest 2010 flare as observed in X-rays (black, $300 \mathrm{~s}$ binning) and in the UV (red, $60 \mathrm{~s}$ binning.)

observed with the same instrumental setup as DN Tau in 2010. Accounting for radius and distance, i.e., enlarging EV Lac to the size of DN Tau and putting it at the same distance, we find that a scaled-up version of an active M dwarf outshines DN Tau by a factor of 1.5-2.0 in X-rays, but would only produce $15 \%$ to $20 \%$ of its UV flux. When scaling these values to DN Tau's true X-ray emission, i.e. accounting for the X-ray overluminosity of the scaled up M dwarf, only about $10 \%$ of the UV flux from DN Tau are attributable to magnetic activity. While a mild suppression in X-ray brightness in accreting vs. nonaccreting $\mathrm{T}$ Tauri stars is quite typical and might be related to phenomena not present on M dwarfs, this comparison shows that the bulk of the UV emission from DN Tau is generated in the accretion shocks.

In contrast, the three X-ray flares observed in 2010 have clear counterparts in the UVW1 data, and the most prominent is the largest flare starting around $55 \mathrm{ks}$. In Fig. 4 we show its UV light curve overplotted with the X-ray light curve, scaled to the same quasi-quiescent preflare level for clarity. The UV emission precedes the X-rays and peaks about 10 min earlier. This behavior indicates an energy release via magnetic reconnection, succeeded by evaporation of fresh material from the stellar surface that is subsequently heated to X-ray emitting temperatures. Flare events like these are frequently observed on the Sun and lowmass stars. Using results from spectral modeling (see Sect. 4.3), we estimate a peak luminosity of $L_{\mathrm{X}}=3 \times 10^{30} \mathrm{erg} \mathrm{s}^{-1}$ for the largest flare, an energy release of about $2.5 \times 10^{33} \mathrm{erg}$ at X-ray energies, and a loop length of about $L \approx 0.15 R_{*}$, i.e. an event in a compact coronal structure located close to the stellar surface of DN Tau. The time evolution of the flare is dominated by the initial event, but shows substructure as visible in the optical plateau and the secondary X-ray peak, indicating subsequent magnetic activity. About one hour after the flare onset, the X-ray and UV light curves roughly reach their preflare values again.

The two smaller X-ray flares show even more complex light curves. They probably result from an overlay of multiple events, for example, several magnetic reconnections occurring within a short time interval in an active region or region complex. Both flares again show UV counterparts, but these are less pronounced than during the large event.

\subsection{Global X-ray properties from CCD spectroscopy}

To study the global spectral properties of the X-ray emission from DN Tau we use the EPIC data. As an example of the spectral quality we show in Fig. 5 the PN spectra and corresponding

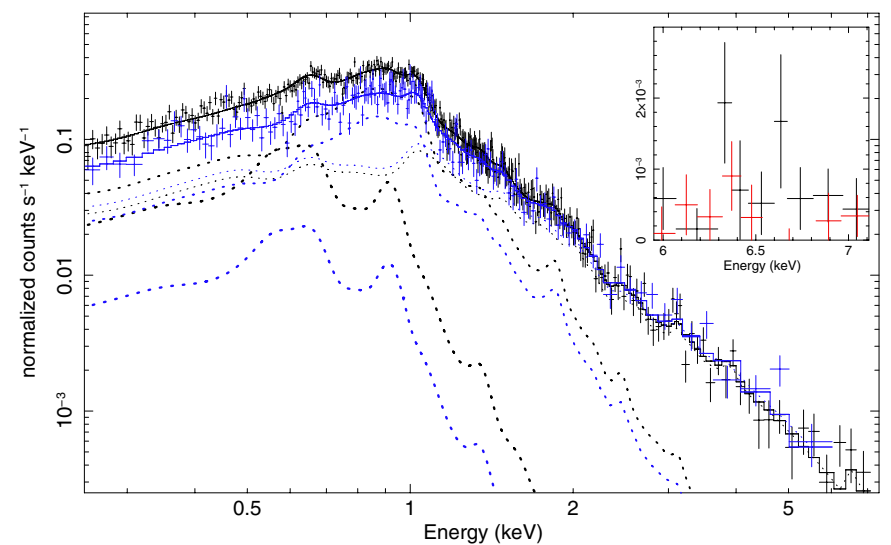

Fig. 5. X-ray spectra of DN Tau (crosses, PN), spectral fits (histograms), and respective model components (dashed) for the two observations: 2010 (black), 2005 (blue). Inset: the spectrum above $6.0 \mathrm{keV}$ during the active (black) and quasi-quiescent (red) half in 2010.

models for the two observations; in the inset, we show the X-ray emission at high energies, which is discussed below. Visual inspection already shows that major changes have occurred in the softer X-ray regime, whereas the spectra above $1 \mathrm{keV}$ are virtually identical. The similar spectral slopes suggest that the changes at low energies are not caused by variable line-of-sight absorption, but are intrinsic to the emission of DN Tau.

The spectral properties of DN Tau and their changes between the two observations are quantified by modeling the spectra in iterative steps to obtain the most robust results. We first investigate potential effects of the flares on the total $2010 \mathrm{spec}-$ trum, but found no differences between the models for the quasiquiescent $(t<55 \mathrm{ks})$ and active half, except for small renormalizations by a few percent. In a next step we fitted the total data of each observation individually. No significant changes in absorption column density and coronal abundances were found between the 2005 and 2010 datasets, and we tied these parameters. We then modeled both observations simultaneously with temperatures and emission measures (EM) as free parameters. We first used the MOS detectors that have better spectral resolution to determine EMDs, abundances, and absorption, and cross-checked our results with the PN data, where we also tied the temperatures. The derived model parameters are given in Table 3, and a comparison shows that the overall coronal temperature structure and the EMD changes are independent of the data used. The X-ray luminosities are the emitted ones; i.e., they are absorption-corrected. (The observed values are given in brackets.) We find for the 2010 (2005) observation X-ray luminosities of $\log L_{\mathrm{X}}=30.2(30.1) \mathrm{erg} \mathrm{s}^{-1}$ average coronal temperatures of $T_{\mathrm{X}}=13$ (18) MK and an activity level $\log L_{\mathrm{X}} / L_{\mathrm{bol}} \approx-3.3$, emphasizing that DN Tau is among the most active and X-ray brightest CTTSs with respect to its mass or effective temperature.

The emission measure distributions show that intermediate ( $\sim 6-8 \mathrm{MK})$ and high $(\gtrsim 20 \mathrm{MK})$ temperature plasma dominates the X-ray emission from DN Tau in both observations, whereas the cool component around $2 \mathrm{MK}$ contributes only about $5 \%$ (2005) and 15\% (2010) to the total emission measure. While the fitted temperatures are comparable between the 2005 and 2010 data for all plasma components, the emission measure of the individual components varies distinctly. We find a strong EM increase by roughly a factor three in the cool component and a moderate increase of $50 \%$ in the intermediate-temperature component. In contrast, a constant EM or even moderate decrease is 
Table 3. Spectral fit results for DN Tau, EPIC data.

\begin{tabular}{|c|c|c|c|}
\hline Par. & 2005 & 2010 & Unit \\
\hline \multicolumn{4}{|c|}{ MOS } \\
\hline$N_{\mathrm{H}}$ & \multicolumn{2}{|c|}{$0.8_{-0.1}^{+0.1}$} & $10^{21} \mathrm{~cm}^{-2}$ \\
\hline kT1 & $0.17_{-0.03}^{+0.05}$ & $0.23_{-0.03}^{+0.03}$ & $\mathrm{keV}$ \\
\hline kT2 & $0.60_{-0.06}^{+0.07}$ & $0.64_{-0.03}^{+0.03}$ & $\mathrm{keV}$ \\
\hline kT3 & $2.27_{-0.21}^{+0.33}$ & $1.91_{-0.14}^{+0.15}$ & $\mathrm{keV}$ \\
\hline EM1 & $0.8_{-0.5}^{+0.9}$ & $2.0_{-0.5}^{+0.6}$ & $10^{52} \mathrm{~cm}^{-3}$ \\
\hline EM2 & $3.7_{-0.4}^{+0.5}$ & $5.6_{-0.6}^{+0.5}$ & $10^{52} \mathrm{~cm}^{-3}$ \\
\hline EM3 & $6.4_{-0.5}^{+0.6}$ & $5.3_{-0.4}^{+0.5}$ & $10^{52} \mathrm{~cm}^{-3}$ \\
\hline $\operatorname{Mg}(7.6 \mathrm{eV})$ & \multicolumn{2}{|c|}{$0.52_{-0.18}^{+0.26}$} & solar \\
\hline $\mathrm{Fe}(7.9 \mathrm{eV})$ & \multicolumn{2}{|c|}{$0.35_{-0.10}^{+0.12}$} & solar \\
\hline $\mathrm{Si}(8.2 \mathrm{eV})$ & \multicolumn{2}{|c|}{$0.32_{-0.12}^{+0.14}$} & solar \\
\hline $\mathrm{S}(10.4 \mathrm{eV})$ & \multicolumn{2}{|c|}{$0.24_{-0.17}^{+0.18}$} & solar \\
\hline $\mathrm{O}(13.6 \mathrm{eV})$ & \multicolumn{2}{|c|}{$0.65_{-0.16}^{+0.28}$} & solar \\
\hline $\mathrm{Ne}(21.6 \mathrm{eV})$ & \multicolumn{2}{|c|}{$1.51_{-0.38}^{+0.49}$} & solar \\
\hline$\chi_{\text {red }}^{2}$ (d.o.f.) & \multicolumn{2}{|c|}{$1.05(432)$} & \\
\hline$L_{\mathrm{X}}(0.2-8.0 \mathrm{keV})$ & $1.37(0.99)$ & $1.64(1.13)$ & $10^{30} \mathrm{erg} \mathrm{s}^{-1}$ \\
\hline \multicolumn{4}{|c|}{ PN } \\
\hline kT1 & \multicolumn{2}{|c|}{$0.24_{-0.03}^{+0.03}$} & $\mathrm{keV}$ \\
\hline kT2 & \multicolumn{2}{|c|}{$0.64_{-0.02}^{+0.02}$} & $\mathrm{keV}$ \\
\hline kT3 & \multicolumn{2}{|c|}{$1.95_{-0.10}^{+0.11}$} & $\mathrm{keV}$ \\
\hline EM1 & $0.5_{-0.3}^{+0.2}$ & $2.0_{-0.3}^{+0.3}$ & $10^{52} \mathrm{~cm}^{-3}$ \\
\hline EM2 & $3.5_{-0.4}^{+0.4}$ & $5.6_{-0.4}^{+0.3}$ & $10^{52} \mathrm{~cm}^{-3}$ \\
\hline EM3 & $5.9_{-0.4}^{+0.3}$ & $5.2_{-0.3}^{+0.4}$ & $10^{52} \mathrm{~cm}^{-3}$ \\
\hline$\chi_{\text {red }}^{2}$ (d.o.f.) & \multicolumn{2}{|c|}{$1.04(508)$} & \\
\hline$L_{\mathrm{X}}(0.2-8.0 \mathrm{keV})$ & $1.24(0.90)$ & $1.62(1.12)$ & $10^{30} \mathrm{erg} \mathrm{s}^{-1}$ \\
\hline
\end{tabular}

Notes. Parameters absent in the PN results are adopted from the MOS modeling.

present in the hot component. In relative terms the EM-increase is most pronounced in the cool component, but in absolute terms the increase in the intermediate temperature component is at least comparable to it or even slightly larger.

The X-ray luminosity of $1.6 \times 10^{30} \mathrm{erg} \mathrm{s}^{-1}$ obtained for the $2010 X M M-N e w t o n$ data is about $25 \%$ higher than those in the XMM-Newton observation from 2005, but a factor of three above the values obtained from Einstein data roughly 30 years ago and from ROSAT data in the early 1990s. Neither the 2005 nor the 2010 exposure are dominated by strong flaring, thus significant, likely long-term, variability of DN Tau's X-ray brightness must be present, and clearly this distinct change has to occur in the emission components associated with magnetic activity.

Our spectral modeling shows that the moderate X-ray brightening is caused by an increase in EM in the cool and intermediate plasma component. These components contribute much more in 2010 to the EMD than in 2005. Typically magnetically more active phases show harder spectra due to the stronger contribution from hotter plasma, but since cooler and hotter coronal regions are not cospatial and significant evolution may have occurred over the five years, a coronal origin for the EMD changes cannot be completely ruled out by the plasma temperatures alone. Given the CTTS nature of DN Tau and because a similar trend, albeit on timescales of hours, was observed on the prototype of an accretion-dominated CTTS TW Hya (Robrade \& Schmitt 2006), another possibility would be to attribute the enhanced emission from cool plasma on DN Tau to the presence of a stronger accretion component. In this scenario the coolest component would naturally be predominantly affected, since here the contribution from the accretion shock is greatest. While there is also a coronal contribution to the low temperature plasma and clearly the $8 \mathrm{MK}$ plasma does not originate directly in the accretion shocks, the EM increase at intermediate temperatures might be a contribution from an accretionally fed coronal component, as suggested by Brickhouse et al. (2010) in their study of TW Hya. That the hot component ( $\gtrsim 20 \mathrm{MK})$, attributed to the corona of DN Tau, stayed approximately constant with a tendency toward a mild decrease again does not favor enhanced magnetic activity as the origin of the increased X-ray brightness. Furthermore, this scenario would imply that the enhanced accretion component had at best a very moderate effect on the hot coronal structures associated with the magnetically most active regions on the surface of DN Tau.

We find an overall low metallicity in the X-ray spectra of DN Tau, but significant differences for individual elemental abundances are present. The derived abundance pattern of DN Tau shows in general a so-called IFIP (inverse first ionization potential) pattern that is commonly observed in active stars, where the low FIP elements like Fe are significantly depleted, and especially the high FIP elements like Ne are enhanced compared to solar composition. The IFIP trend is not strictly linear in DN Tau (see Table 3 where the FIP of each element is given in brackets), but appears to be have a broad abundance minimum at low-to-intermediate FIP elements (Fe-Si-S), while the very low FIP element $\mathrm{Mg}$ and the intermediate FIP element $\mathrm{O}$ have higher abundances and only $\mathrm{Ne}$ is enhanced compared to solar photospheric values. While the absolute abundances vary moderately between the applied models, the derived abundance ratios are fairly robust. Independent of the specific model or data used, our best fits give a $\mathrm{Ne} / \mathrm{O}$ ratio, as well as a $\mathrm{O} / \mathrm{Fe}$ ratio, of roughly two for DN Tau, similar to values observed for BP Tau (Robrade \& Schmitt 2006) and in many active M dwarf coronae (Güdel et al. 2001; Robrade \& Schmitt 2005).

\subsubsection{The spectrum beyond $6 \mathrm{keV}$}

In the inset of Fig. 5 we show the X-ray emission from DN Tau at very high energies; here the PN spectra above $6.0 \mathrm{keV}$ from the 2010 observation roughly split in the middle and binned to a minimum of five counts. The comparison shows that photons at these energies were predominantly collected during the second and more active half of the observation, defined as $t>55 \mathrm{ks}$. We identify probable contributions from the $6.4 \mathrm{keV} \mathrm{Fe}-\mathrm{K} \alpha$ fluorescence line, which is excited by photons with energies above $7.1 \mathrm{keV}$, from the $6.7 \mathrm{keV} \mathrm{Fe} \mathrm{XXV} \mathrm{line}$ complex and possibly also from the $6.97 \mathrm{keV}$ Fe XXVI line. When adding a narrow Gaussian at $6.4 \mathrm{keV}$ to the $2010 \mathrm{spec}-$ tral model, where fluorescence photons were not included, we find that the $\mathrm{Fe}-\mathrm{K} \alpha$ line is formally detected, but its flux is with $2.1(0.3-3.9) \times 10^{-15} \mathrm{ergs} \mathrm{cm}^{-2} \mathrm{~s}^{-1}$ poorly defined. The additional presence of emission lines from highly ionized $\mathrm{Fe}$ indicates that plasma with temperatures of $\gtrsim 40 \mathrm{MK}$ is generated in active structures on DN Tau, most likely predominantly during the detected flares. Nevertheless, while the spectra clearly suggest the presence of very hot plasma on DN Tau, especially in the more active half of the 2010 observation, even at this phase its contribution to the total X-ray emission is, with a few percentage points, very minor. 
Table 4. Line fluxes in $10^{-5} \mathrm{cts}^{-2} \mathrm{~s}^{-1}$, absorption-corrected.

\begin{tabular}{ccccc}
\hline \hline Data & Ly $\alpha$ & $r$ & $i$ & $f$ \\
\hline & OVIII & OVII \\
\hline 2010 & $4.0 \pm 0.5$ & $1.4 \pm 0.4$ & $1.4 \pm 0.5$ & $0.5 \pm 0.3$ \\
2005 & $2.3 \pm 1.2$ & $0.7 \pm 0.5$ & $1.3 \pm 0.7$ & $1.2 \pm 0.7$ \\
\hline \multicolumn{5}{c}{ Ne IX } \\
\hline 2010 & $1.6 \pm 0.2$ & $0.8 \pm 0.2$ & $0.2 \pm 0.2$ & $0.7 \pm 0.2$ \\
\hline
\end{tabular}

\subsubsection{X-ray absorption towards DN Tau}

Absorption can significantly alter the appearance of X-ray spectra and we derive a moderate absorption column density of $N_{\mathrm{H}}=0.8 \times 10^{21} \mathrm{~cm}^{-2}$ from our modeling, showing that no large amounts of circumstellar or disk material are in the line of sight. The X-ray absorption is, in contrast to extinction, also sensitive to optically transparent material, and thus it is a useful tool for studying infalling or outflowing dust-free gas or plasma. As mentioned above, the modeled X-ray absorption is virtually unaffected by the observed changes in the EMD. Consequently, if the cooler X-ray plasma is largely created in the vicinity of the accretion shocks and the increase in emission measure is caused by a higher mass accretion rate, then the plasma in the accretion columns can make at most a very moderate contribution to the modeled X-ray absorption in DN Tau. The X-ray absorption of DN Tau is generally consistent with the one expected from the optical extinction $A_{V} \approx 0.3 \ldots 0.5 \mathrm{mag}$, when using the standard conversion $N_{\mathrm{H}}=1.8 \times 10^{21} \mathrm{~cm}^{-2} \times A_{V} \mathrm{~cm}^{-2}$ (Predehl \& Schmitt 1995). When adopting a roughly standard gas-to-dust ratio, an extinction of $A_{V}=0.9$ as used by Ingleby et al. (2013) is not supported by the X-ray results. Several other CTTSs (e.g., BP Tau) also show agreement within a factor of two between X-ray and optical absorption. In contrast, the more pole-on CTTS RU Lup (Robrade \& Schmitt 2007) or the near edge-on system AA Tau (Schmitt \& Robrade 2007) exhibit an X-ray absorption that is up to about one magnitude above the values derived from optical measurements, and indeed most CTTSs show an excess X-ray absorption in Günther \& Schmitt (2008). This finding indicates that mainly matter with a "normal", i.e. roughly interstellar, gas-to-dust ratio is responsible for the absorption towards the X-ray emitting regions on DN Tau. Significant amounts of optically transparent material such as accretion streams or hot winds are absent in the line of sight, probably favored by the fact that DN Tau is viewed under an intermediate inclination.

\subsection{High-resolution X-ray spectroscopy}

The high-resolution RGS spectrum of DN Tau obtained in 2010 is shown in Fig. 6, here flux-converted in the 8-25 $\AA$ range. A global modeling of these data leads to overall similar results as derived above, and we concentrate in the following on the analysis of the brighter emission lines denoted in the plot. These density and temperature sensitive lines are of special diagnostic interest, since they can be used to study the plasma contributions originating in the corona and accretion spots.

In our analysis we use the lines of the He-like triplet of $\mathrm{O}$ VII, namely resonance $(r)$, intercombination $(i)$, and forbid$\operatorname{den}(f)$ at 21.6, 21.8, and 22.1 $\AA$, as well as the Ly $\alpha$ line of O VIII at $18.97 \AA$. The absorption-corrected photon fluxes of the relevant lines, using the $N_{\mathrm{H}}$ value from our EPIC modeling, are given in Table 4, and a zoom on the O VII triplet for the two exposures is shown in Fig. 7. We also make a comparison to

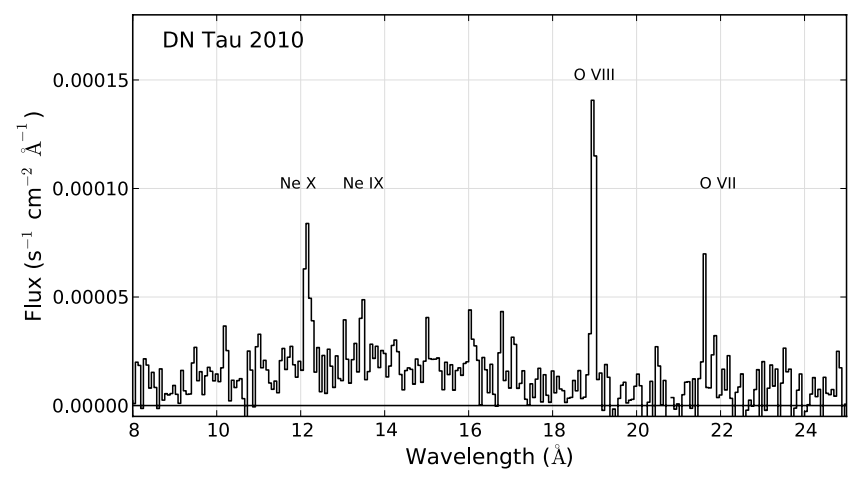

Fig. 6. Flux-converted RGS spectrum (2010 observation) of DN Tau.

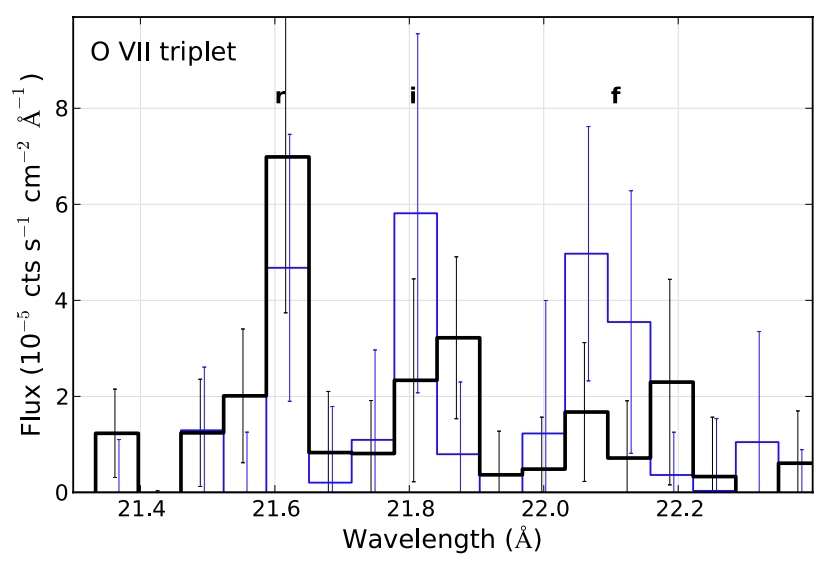

Fig. 7. Observed O VII triplet in 2010 (black) and 2005 (blue).

the 2005 observation to check our results from the global spectroscopy, but admittedly the $\mathrm{S} / \mathrm{N}$ of these data is rather poor. A similar diagnostic for moderately hotter plasma uses the Ne IX triplet $(13.45-13.7 \AA)$ and the Ne X line $(12.1 \AA)$, which are detected in the 2010 spectrum. These lines allow also an abundance analysis of the $\mathrm{Ne} / \mathrm{O}$ ratio when applying the methods described in Robrade et al. (2008). For DN Tau we find $\mathrm{Ne} / \mathrm{O} \approx 0.4$, a typical value for an active star and similar to the one derived above.

\subsubsection{Oxygen lines - plasma density}

To search for high-density plasma from accretion shocks, we specifically study the density sensitive $f / i$-ratio of the O VII triplet (see, e.g., Porquet et al. 2001), which has a peak formation temperature of about $2 \mathrm{MK}$.

The plasma density is determined from the relation $f / i=$ $R_{0} /\left(1+\phi / \phi_{\mathrm{c}}+n_{\mathrm{e}} / N_{\mathrm{c}}\right)$ with $f$ and $i$ the respective line intensities, $R_{0}=3.95$ the low density limit of the line ratio, $N_{\mathrm{c}}=$ $3.1 \times 10^{10} \mathrm{~cm}^{-3}$ the critical density, and $\phi / \phi_{\mathrm{c}}$ the radiation term. The effect from radiation is neglected in our calculations since the UV field of DN Tau is not strong enough to influence the $\mathrm{O}$ VII ratio. A strong FUV flux would lower the derived plasma densities, but in the case of DN Tau, the FUV emission would also to be attributed to the accretion shocks, which however only produce a rather small UV excess. On the other hand, O VII is not only produced in the accretion shocks, but also in the corona, which is dominated by low-density plasma, and the true accretion shock density would be underestimated. As a consequence, changes in the measured O VII density can be caused either by changes in the actual densities in the accretion components or 
by the relative mixture of low- and high-density plasma from the corona and the accretion shocks.

As shown in Fig. 7, the O VII intercombination line is stronger than the forbidden line in the 2010 spectrum, while in the 2005 data they are of comparable strength. We find a $f / i$-ratio below one in both observations; the derived values are $f / i=0.36 \pm 0.26$ for the 2010 data and $f / i=0.92 \pm 0.73$ for the 2005 data from measured line fluxes. Poissonian ranges (90\% conf.) derived from Monto-Carlo methods on the measured counts are 0.14-0.62 (2010) and 0.06-2.0 (2005). Coronal sources typically exhibit a higher ratio of $f / i \gtrsim 1.5$ (Ness et al. 2004), indicating the presence of noncoronal plasma on DN Tau. The $f / i$-ratio differs by a factor of 2.5 between the observations, but large errors, especially for the 2005 exposure, are present. For the $\mathrm{O}$ VII emitting plasma, we find a density of $n_{\mathrm{e}}=3.0(1.6-11.8) \times 10^{11} \mathrm{~cm}^{-3}(2010)$ and $n_{\mathrm{e}}=1.0(0.4-6.1) \times$ $10^{11} \mathrm{~cm}^{-3}$ (2005), respectively; given the coronal contribution, these values are likely lower limits for the accretion shocks. Overall, the densities derived for DN Tau are comparable, but at the lower end of values found for other CTTSs. Given a theoretical "low density" $f / i$-ratio of around four, the coronal contribution at $\mathrm{O}$ VII temperatures is expected to be only moderate, and an inspection of our spectral model shows that virtually all ( $\sim 90 \%)$ of the O VII emission is generated by the coolest plasma component. An apparent higher density in 2010 would be explained naturally by a stronger contribution of accretion plasma to the X-ray emission, either owing to a larger spatial extent or to a higher density of the shock region(s).

Inspecting the $f / i$-ratio for high densities, i.e. $\log n_{\mathrm{e}} \gtrsim$ $12 / 13 \mathrm{~cm}^{-3}$, one finds $f / i \lesssim 0.1 / 0.01$. Thus the O VII triplet is at the very end of its density-sensitive range, and even small contributions from the omnipresent corona can strongly affect the results. For example, adding to a high-density plasma with $\log n_{\mathrm{e}} \sim$ $13 \mathrm{~cm}^{-3}$, a $10 \%$ fraction of coronal material $(f / i \sim 3)$ already reduces the apparent density by about one order of magnitude. In these cases the O VII analysis does not measure the true density of the accretion shock or the average density of the stellar plasma, but primarily traces the relative contributions from the visible portions of the accretion shocks and corona. Assuming shocks with high density and a corona with low density, we derive an accretion to coronal EM-ratio of about $0.7: 1$ in 2005 that increased to a ratio of 2:1 in the year 2010. Correspondingly, one would expect, when assuming a similar corona, a comparable increase in the coolest plasma component, and while there appears to be some deficit in total O VII photons, this is roughly consistent with our finding from $\mathrm{O}$ VII $(r)$ and the EMD modeling.

A density analysis of hotter plasma at $\approx 4 \mathrm{MK}$ can be carried out with the Ne IX triplet, which also has a higher critical density. Here we find a $f / i$-ratio that is compatible with its low-density limit $\left(n_{\mathrm{e}} \approx 1.0 \times 10^{11} \mathrm{~cm}^{-3}\right)$, while at the high end we can only put an upper limit of $n_{\mathrm{e}} \lesssim 5 \times 10^{12} \mathrm{~cm}^{-3}$ on the plasma density. In addition, the Ne IX $i$ line is blended by several Fe lines with Fe XIX being the strongest one. This is not taken into account in the above calculation since Fe is heavily depleted in DN Tau, and statistical errors on the only weakly detected $i$-line dominate. Moderately cooler plasma at $\sim 1.5 \mathrm{MK}$ could be studied with the $\mathrm{N}$ VI triplet, but the data quality is insufficient to provide any further constraints.

\subsubsection{Oxygen lines - plasma temperatures}

Accretion processes that contribute to the X-ray emission can also be studied with temperature diagnostics. The material that is accreted by CTTSs has infall velocities of a few hundred $\mathrm{km} \mathrm{s}^{-1}$

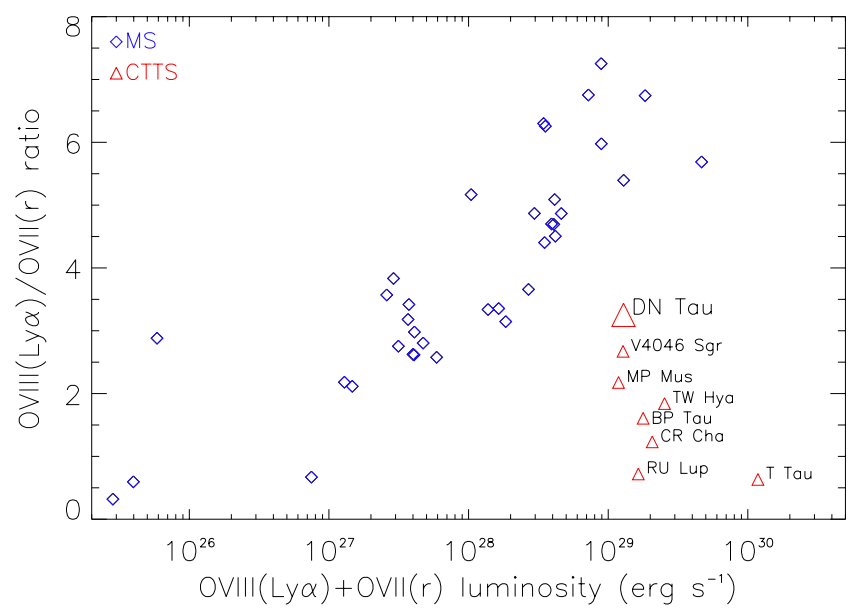

Fig. 8. The soft excess of DN Tau; O VIII $\left(\operatorname{Ly}_{\alpha}\right) / \mathrm{O}$ VII(r)-ratio vs. summed luminosity for main-sequence stars (diamonds) and CTTSs (triangles).

and thus the post-shock plasma reaches at maximum temperatures up to a few MK. Such plasma is still relatively cool with respect to the coronal temperatures in active stars or CTTSs and should therefore be detectable as "soft-excess" X-ray emission. Here we search for an excess of cool plasma via the $\mathrm{O}$ VIII/O VII line ratio.

As temperature diagnostic we use strong oxygen emission lines as measured in 2010 , here the $\mathrm{O}$ VIII Ly $\mathrm{Ly}_{\alpha}$ line $(18.97 \AA)$ and the O VII He-like triplet lines with peak formation temperatures of $\sim 3 \mathrm{MK}$ and $\sim 2 \mathrm{MK}$, respectively. An abundance-independent method is obtained by using the $\mathrm{O}$ VIII( $\left(\mathrm{Ly}_{\alpha}\right) / \mathrm{O}$ VII(r) energy flux ratio in comparison to the summed luminosity of both lines.

In Fig. 8 we compare the $\mathrm{O}$ VIII/O VII-ratio of DN Tau with those of other CTTSs collected in the literature and with a large sample of main-sequence stars at various activity levels taken from Ness et al. (2004). The correlation between the O VIII/O VII line ratio and $L_{X}$ for main-sequence stars is well known and caused by the higher coronal temperatures in more active and X-ray brighter stars. As shown in the plot, DN Tau exhibits a soft excess compared to active coronal sources with similar X-ray luminosity, but it is quite weak when compared to other CTTSs. Actually the soft excess of DN Tau is the weakest in the sample of X-ray studied CTTSs. Calculating the O VIII/O VII energy flux ratio of DN Tau, we find a value of around three. When inspecting theoretical ratios as calculated with, say, the Chianti code, this corresponds to an average plasma temperature of 3.0-3.5 MK. This temperature is rather high given the expected shock temperatures and supports the idea that most of the oxygen emission in DN Tau is produced by magnetic activity or consists of mixed accreted and coronal plasma. Another method based on O VII alone, and thus more suited for very cool temperatures, uses the temperature sensitive g-ratio, $g=(f+i) / r$. Our value of $g=1.36 \pm 0.57$ indeed favors low temperatures of $\lesssim 1 \mathrm{MK}$ for the O VII plasma, but owing to its weaker temperature dependence, twice as high temperatures are also consistent with the data.

\section{Discussion}

\subsection{Accretion shocks on DN Tau}

At first glance, the soft excess of DN Tau is surprisingly weak for a young CTTS that is accreting matter from its disk and that exhibits a high plasma density in O VII. Two main factors might 
be responsible for this effect: either the relative accretion luminosity is very low or the accreted plasma is not heated enough to produce strong O VII emission.

The estimated mass accretion rates from optical/UV observations of DN Tau are intermediate for CTTSs, so that they cannot alone explain the weakness of its soft excess. Here the ratio of coronal-to-accretion luminosity is another important measure. While the density analysis suggests a significant contribution of the accretion shock to the O VII emission, the relative contribution from very cool plasma to the overall X-ray emission is quite weak, and a strong $\mathrm{O}$ VIII line from the corona reduces the soft excess. Also, the evolutionary phase plays an important role, and DN Tau is a low-mass CTTS that is relatively young and thus still quite enlarged. As a consequence, shock speeds and temperatures do not reach values found for more massive or older, more compact stars. Basically, $V_{\mathrm{sh}} \propto \sqrt{2 G M_{*} / R_{*} \times\left(1-R_{*} / R_{\mathrm{t}}\right)}$ and $T_{\mathrm{sh}} \propto V_{\mathrm{sh}}^{2}$ (e.g. Lamzin 1998; Calvet \& Gullbring 1998), where $M_{*}$ and $R_{*}$ are stellar mass and radius and $R_{\mathrm{t}}$ is the disk truncation radius, i.e., from where matter is falling onto the star.

The calculated shock velocity depends slightly on the adopted stellar model, using the Donati et al. (2013) values of $M_{*}=0.65 M_{\odot}$ and $R_{*}=1.9 R_{\odot}$, and their $R_{\mathrm{mag}}=5.9 R_{*}$ (2010) as truncation radius results in shock speeds of about $V_{\mathrm{sh}}=330 \pm 30 \mathrm{~km} \mathrm{~s}^{-1}$. Adopting the slightly larger or less massive stellar models with, say, $M_{*} \approx 0.5 M_{\odot}$ and $R_{*} \approx 2.1 R_{\odot}$ gives $V_{\mathrm{sh}}=260-300 \mathrm{~km} \mathrm{~s}^{-1}$. Here we assumed accretion from the respective corotating radius. The corresponding strong-shock temperatures are in the range of $0.9-1.5 \mathrm{MK}$ and similar to the $\mathrm{O}$ VII-temperature derived above. While these temperatures are sufficient to produce O VII emission, they are below the peak emissivity temperature of about $2 \mathrm{MK}$, reducing the contribution from the accretion shocks to these lines and consequently the strength of the soft excess. Since the hot and active corona emits predominantly at higher temperatures, it contributes even more weakly to O VII and thus preserves the accretion shock signatures in the applied emission line diagnostic, most prominently seen in the very cool plasma during DN Tau's 2010 soft state. However, compared to other CTTSs, the "pure" accretion shock plasma is a weak contributor to the total X-ray emission on DN Tau.

The X-ray mass accretion rate for DN Tau can be calculated from mass conversation under the assumption of a strong shock by using $\dot{M}_{\text {acc }}=4 \pi f R_{*}^{2} \rho_{\text {pre }} V_{\text {sh }}$. Using our best-fit modeling results on the plasma density and adopting a mean molecular weight of $\mu_{\mathrm{e}}=1.2$, a filling factor of $f=0.01$, and stellar models as above, we derive an X-ray mass accretion rate of $\log \dot{M} \approx-9.5 M_{\odot} \mathrm{yr}^{-1}$. As a caveat and recalling the discussion above, the coronal blend means that the OVII density is very likely a lower limit for the accretion shock density and the filling factor is adopted from other analysis. An independent estimate based on X-ray data can be derived when fitting our X-ray spectra with the model from Günther et al. (2007), where we obtain a mass accretion rates around $\log \dot{M}=-9.2 M_{\odot} \mathrm{yr}^{-1}$. With these models we find filling factors of $f \lesssim 0.01$ and post-shock densities of $n_{\mathrm{e}} \gtrsim 3 \times 10^{11} \mathrm{~cm}^{-3}$, but their interdependency does not allow the accretion shock properties to be constrained further. The X-ray accretion rate is about one order of magnitude below the values mostly found from optical/UV measurements, similar to results obtained for other CTTSs (e.g. BP Tau, Schmitt et al. 2005). While intrinsic variability probably also plays a role, this finding might indicate that the accreted material contributes only fractionally to the observed X-ray emission.

In these scenarios, either not all accreted material produces $\mathrm{X}$-rays or the X-rays are produced but partially absorbed or both.
In some accretion regions, the shock temperatures might be too low to generate X-ray emission, so X-rays would trace only the fastest fraction of the accretion stream, although it remains unclear why infalling material should not impact the stellar surface with similar velocities when accreted from similar distances, i.e. around the disk truncation radius. Similarly, accretion streams of low density that produce no strong X-ray emission may remain mostly undetected and lead to missing material, but if they exist they are expected to carry only a small fraction of the total mass flux. For example, adding low flux columns $\left(F \propto \rho V^{3}\right)$ to the model of DN Tau, increases the maximum spot size $(f)$ by a factor of 30 , but the mass accretion rate $(\dot{M})$ only by less than a factor of two (Ingleby et al. 2013). Alternatively, virtually all infalling material might produce $\mathrm{X}$-ray emission in accretion shocks, but these X-rays are partly absorbed locally, for example, by the accretion column and thus missing in the observed X-ray spectra as suggested by Sacco et al. (2010).

Recently, Dodin et al. (2013) have performed a non-LTE modeling of emission components of optical $\mathrm{He}$ and $\mathrm{Ca}$ lines by adding an accretion hot spot and a photosphere. Significant variability is present in the derived accretion parameters for two DN Tau observations performed in Oct. 2009 and March 2010. They find preshock (infall) densities of $\log n_{\mathrm{e}}=12.2 / 13 \mathrm{~cm}^{-3}$, velocities of $V_{0}=230 / 280 \mathrm{~km} \mathrm{~s}^{-1}$, and filling factors of $f=$ $3 / 1.2 \%$, leading to accretion luminosities of $3 / 16 \% L_{*}$ and mass accretion rates of $\log \dot{M}=-8.1 /-7.6 M_{\odot} \mathrm{yr}^{-1}$. These results again indicate a very high infall density, high mass accretion rates, and large filling factors for DN Tau. Besides that, they support an active accretion period in 2010, at least a few months before the new X-ray data was taken. where a strong accretion stream impacts high- latitude regions. The 2010 data presented in Donati et al. (2013) was obtained a few months after our X-ray observation and although they obtained a lower mass accretion rate of $\log \dot{M}=-9.1 M_{\odot} \mathrm{yr}^{-1}$, their surface maps show a large monolithic dark spot and an embedded accretion region at similar high latitudes. Their dominant spot is located in 2010 at about phase 0.55 , and our X-ray data was taken at phase $0.1-0.3$. Thus if this configurations is applicable, it involves a relatively unspoiled view on the accretion spot region during the XMM-Newton observation.

In summary, the low infall velocities and the non-negligible coronal contribution likely make X-ray diagnostics less favorable for a quantitative analysis, but they are still applicable to detect the presence of X-rays from accretion shocks in CTTSs like DN Tau.

\subsection{X-ray variability}

The observed X-ray variability can be caused by intrinsic changes in accretion rate or magnetic activity, as well as by varying the viewing geometry, absorption, and rotation. While all these factors contribute to the omnipresent short- and midterm variability (seconds to months), the situation is less clear for the major cause of possible long-term trends on timescales of years to decades. The changes in X-ray brightness between 2005 and 2010 can probably be attributed to different accretion states, unless a large fraction of the accretion spots are "hidden" in the 2005 exposure. Here, variable mass accretion rates and changing magnetic topology, which influences the disk truncation radius and viewing geometry, probably play the main role. Since the X-rays from the accretion shocks experience absorption by the above accretion streams, some timedependent viewing geometry effects may be present in the detected X-ray emission, as suggested in Argiroffi et al. (2011) for 
their V2129 Oph data. Our 2010 observation covers 0.22 in rotational phase and the line of sight is most likely not aligned with the accretion stream, but the 2005 data (0.06 phase coverage) could be more severely affected. While the global $N_{\mathrm{H}}$ is identical for both observations and the apparent presence of highdensity plasma in the 2005 spectrum, as well as for the large EM changes over a broad temperature range, does not strongly support this explanation for the case of DN Tau, the presence of a variable contribution from locally produced and re-absorbed accretion components is not ruled out by the data.

In contrast, the increase in X-ray brightness by a factor of three compared to the measurements in the 1980s and 1990s seems to favor a change in the magnetic activity state of DN Tau, already because its X-ray emission detected by XMM-Newton is predominantly of coronal origin. On the other hand, DN Tau showed on average a brightening over the last decades in the $U$ band, again by a factor of three, probably attributable predominantly to the accretion shocks. These apparently different scenarios might at least be explained partially by an accretion-fed corona that would lead to the observed trends, but unfortunately most of these observations are not simultaneous, and especially $\mathrm{X}$-ray data is sparse. Thus there might be no overall long-term trend at all, but instead UV bright and X-ray bright phases that are not necessarily related to each other and dominated by the various kinds of short-term variability.

\subsection{DN Tau in the CTTS context}

The young low-mass CTTS DN Tau shows a soft excess and a high density in its cool ( $2 \mathrm{MK})$ plasma component. Both findings indicate that plasma originating in well-funnelled accretion streams impacting the stellar surface contributes to the observed $\mathrm{X}$-ray emission. The plasma densities of DN Tau derived from O VII diagnostics are similar to values of other CTTSs, such as BP Tau or RU Lup (Schmitt et al. 2005; Robrade \& Schmitt 2007), but its soft X-ray excess is quite weak for a young CTTS. The higher mass accretion rates of BP Tau or RU Lup naturally lead to a more pronounced soft excess, but this effect cannot account for all CTTSs. Additionally, as a consequence of the low mass and large radius of DN Tau, the impact velocity is among the lowest of all studied CTTSs, and expected accretion shock temperatures are well below the peak formation temperature of the studied X-ray lines. Therefore only the hottest part of the accreted and shocked material will reach X-ray temperatures; however, this is still sufficient to produce detectable signatures in the $\mathrm{X}$-ray data. Combining the only moderate mass accretion rate and the low impact velocity would explain why the soft excess of DN Tau is even less than those of old CTTSs, such as V4046 Sgr or MP Mus. While their accretion rates are even lower, these objects are more massive and especially more compact $\left(M_{*} / R_{*}\right)$, and the accretion shocks have higher temperatures and produce $\mathrm{X}$-ray emission in the O VII-lines more efficiently.

Comparing DN Tau to other young stars in the lower mass regime, we find that a moderate soft excess is also present in the TWA member Hen 3-600 (Huenemoerder et al. 2007), a multiple system with an M3/M3.5 binary as principle components. The value of its O VIII/O VII ratio is similar to the one of DN Tau, although Hen 3-600 is about a factor five fainter in $\mathrm{X}$-rays, and its soft excess is even less pronounced. In contrast to DN Tau, Hen 3-600 is old ( $\sim 10 \mathrm{Myr})$ and very likely already in the CTTS/WTTS transitional phase. While expected to be compact, here the evolved state and corresponding very low accretion rate reduces accretion shock signatures in its X-ray spectrum. Correspondingly, its oxygen $f / i$-ratio of about 1.1 , though with significant error, is at the uppermost end of the values observed for accretional sources.

Notably, coronal properties as derived from the modeling of global X-ray spectra are very similar for DN Tau and BP Tau. We find similar X-ray luminosities and plasma temperatures, as well as nearly identical abundance patterns. The largest differences are found in the relative strength of the cool component of their EMDs, but as outlined above, this is where the accretion shocks have their strongest impact on the X-ray spectra. Overall, at least when considering stars with comparable X-ray activity, the coronal properties seem to vary at best moderately in the regime of young low-mass CTTSs when going to less massive stars.

With DN Tau we extend the X-ray studied sample of young accreting stars to lower masses, and its X-ray properties clearly link it to more massive or more evolved CTTSs. The combination of a very cool accretion component with a strong hot corona makes DN Tau one of the X-ray brightest CTTSs in its mass range, but reduces the influence of the accretion shocks in its $\mathrm{X}$-ray spectrum and emission line diagnostics.

\section{Summary and conclusions}

From our study of the X-ray emission of DN Tau we obtain the following main results and draw the subsequent conclusions.

1. DN Tau is among the least massive CTTSs where cool MK-temperature plasma at high density from accretion shocks is clearly present, and it is the youngest star in the regime of M-type stars studied in X-rays in greater detail. DN Tau shares general properties with other low-mass CTTSs, but differences arise in detail that are mainly related to its youth and low mass.

2. The O VII triplet shows an $f / i$-ratio of about 0.4 , attributed to accretion shocks that contribute significantly to the soft $\mathrm{X}$-ray emission. The corresponding plasma density is $n_{\mathrm{e}}=$ $3-4 \times 10^{11} \mathrm{~cm}^{-3}$, and owing to the coronal contribution, this is likely a lower limit for the accretion shocks. DN Tau shows a soft excess as measured in O VIII/O VII-ratios, confirming the presence of accretion shock plasma. While the plasma density is quite typical when compared to other CTTSs, the soft X-ray excess is rather weak. Here the low impact velocity of the accreted material, a consequence of the low mass and large radius of DN Tau, results in shock temperatures of about 1.0-1.5 MK, well below peak formation of O VII. Overall, the cool plasma component around 2 MK contributes to the X-ray emission only moderately.

3. A strong coronal component with hot ( $\gtrsim 10 \mathrm{MK})$ plasma is present, and at higher energies, the spectrum of DN Tau is dominated by magnetic activity. Intermediate temperature plasma clearly originates in coronal structures, but may contain accretion-fed material. The corona reaches temperatures of $\gtrsim 30 \mathrm{MK}$, and its abundance pattern shows an IFIP effect that is reminiscent of those of active stars. DN Tau is, with $\log L_{\mathrm{X}}=30.2 \mathrm{erg} \mathrm{s}^{-1}$, among the X-ray brighter CTTSs in its mass- or $T_{\text {eff }}$-range.

4. We find significant changes in DN Tau's X-ray properties. In 2010 it was in an X-ray-brighter, but overall softer spectral state compared to 2005. The emission measure of the cool plasma changed by a factor of a few, indicating accretion related variability. Similar, but less pronounced changes are observed at intermediate temperatures; in contrast, the hot component stayed virtually constant. No changes in absorption column or elemental abundances were found; furthermore, the X-ray absorption is consistent with optical values. 
5. Several X-ray flares with durations of $\lesssim 1 \mathrm{~h}$ are detected in the 2010 exposure, which are accompanied by clear UV counterparts. The UV emission precedes the X-rays as expected in the chromospheric evaporation scenario, and the flares are similar to the ones seen on active young $\mathrm{M}$ dwarfs. Outside the observed flares, an obvious correlation between $\mathrm{X}$-ray and UV brightness is not observed, indicating largely independent emission regions. Brightness differences by a factor of three are present in X-ray and $U$ band data on timescales of years to decades.

Acknowledgements. This work is based on observations obtained with XMMNewton, an ESA science mission with instruments and contributions directly funded by ESA Member States and the USA (NASA). J.R. acknowledges support from the DLR under grant 50QR0803. H.M.G. was supported by the National Aeronautics and Space Administration under Grant No. NNX11AD12G issued through the Astrophysics Data Analysis Program. The publication is supported by the Austrian Science Fund (FWF).

\section{References}

Andrews, S. M., \& Williams, J. P. 2005, ApJ, 631, 1134

Argiroffi, C., Maggio, A., \& Peres, G. 2007, A\&A, 465, L5

Argiroffi, C., Flaccomio, E., Bouvier, J., et al. 2011, A\&A, 530, A1

Argiroffi, C., Maggio, A., Montmerle, T., et al. 2012, ApJ, 752, 100

Arnaud, K. A. 1996, in Astronomical Data Analysis Software and Systems V, eds. G. H. Jacoby, \& J. Barnes (San Francisco: ASP), ASP Conf. Ser., 101, 17

Bouvier, J., Bertout, C., \& Bouchet, P. 1986, A\&A, 158, 149

Brickhouse, N. S., Cranmer, S. R., Dupree, A. K., Luna, G. J. M., \& Wolk, S. 2010, ApJ, 710, 1835

Calvet, N., \& Gullbring, E. 1998, ApJ, 509, 802

Cohen, M., \& Kuhi, L. V. 1979, ApJS, 41, 743

Dodin, A. V., Lamzin, S. A., \& Sitnova, T. M. 2013, Astron. Lett., 39, 315

Donati, J.-F., Gregory, S. G., Alencar, S. H. P., et al. 2013, MNRAS, 436, 881

Fernandez, M., Ortiz, E., Eiroa, C., \& Miranda, L. F. 1995, A\&AS, 114, 439

Furlan, E., Luhman, K. L., Espaillat, C., et al. 2011, ApJS, 195, 3

Getman, K. V., Flaccomio, E., Broos, P. S., et al. 2005, ApJS, 160, 319

Grankin, K. N., Melnikov, S. Y., Bouvier, J., Herbst, W., \& Shevchenko, V. S. 2007, A\&A, 461, 183

Grevesse, N., \& Sauval, A. J. 1998, Space Sci. Rev., 85, 161

Güdel, M., \& Telleschi, A. 2007, A\&A, 474, L25

Güdel, M., Audard, M., Magee, H., et al. 2001, A\&A, 365, L344

Güdel, M., Skinner, S. L., Briggs, K. R., et al. 2005, ApJ, 626, L53

Güdel, M., Briggs, K. R., Arzner, K., et al. 2007a, A\&A, 468, 353

Güdel, M., Skinner, S. L., Mel'Nikov, S. Y., et al. 2007b, A\&A, 468, 529
Gullbring, E., Hartmann, L., Briceno, C., \& Calvet, N. 1998, ApJ, 492, 323

Günther, H. M., \& Schmitt, J. H. M. M. 2008, A\&A, 481, 735

Günther, H. M., Liefke, C., Schmitt, J. H. M. M., Robrade, J., \& Ness, J.-U. 2006, A\&A, 459, L29

Günther, H. M., Schmitt, J. H. M. M., Robrade, J., \& Liefke, C. 2007, A\&A, 466, 1111

Hartmann, L., \& Stauffer, J. R. 1989, AJ, 97, 873

Herbig, G. H., \& Bell, K. R. 1988, Third Catalog of Emission-Line Stars of the Orion Population : $3: 1988$

Huenemoerder, D. P., Kastner, J. H., Testa, P., Schulz, N. S., \& Weintraub, D. A. 2007, ApJ, 671, 592

Ingleby, L., Calvet, N., Herczeg, G., et al. 2013, ApJ, 767, 112

Johns-Krull, C. M. 2007, ApJ, 664, 975

Kashyap, V., \& Drake, J. J. 2000, Bull. Astron. Soc. Ind., 28, 475

Kastner, J. H., Huenemoerder, D. P., Schulz, N. S., Canizares, C. R., \& Weintraub, D. A. 2002, ApJ, 567, 434

Kenyon, S. J., \& Hartmann, L. 1995, ApJS, 101, 117

Koenigl, A. 1991, ApJ, 370, L39

Lamzin, S. A. 1998, Astron. Rep., 42, 322

Mitra-Kraev, U., Harra, L. K., Güdel, M., et al. 2005, A\&A, 431, 679

Muzerolle, J., Calvet, N., Hartmann, L., \& D’Alessio, P. 2003, ApJ, 597, L149

Ness, J.-U., \& Wichmann, R. 2002, Astron. Nachr., 323, 129

Ness, J.-U., Güdel, M., Schmitt, J. H. M. M., Audard, M., \& Telleschi, A. 2004, A\&A, 427, 667

Neuhäuser, R., Sterzik, M. F., Schmitt, J. H. M. M., Wichmann, R., \& Krautter, J. 1995, A\&A, 297, 391

Nguyen, D. C., Jayawardhana, R., van Kerkwijk, M. H., et al. 2009, ApJ, 695, 1648

Porquet, D., Mewe, R., Dubau, J., Raassen, A. J. J., \& Kaastra, J. S. 2001, A\&A, 376, 1113

Predehl, P., \& Schmitt, J. H. M. M. 1995, A\&A, 293, 889

Robrade, J., \& Schmitt, J. H. M. M. 2005, A\&A, 435, 1073

Robrade, J., \& Schmitt, J. H. M. M. 2006, A\&A, 449, 737

Robrade, J., \& Schmitt, J. H. M. M. 2007, A\&A, 473, 229

Robrade, J., Schmitt, J. H. M. M., \& Favata, F. 2008, A\&A, 486, 995

Sacco, G. G., Orlando, S., Argiroffi, C., et al. 2010, A\&A, 522, A55

Schmitt, J. H. M. M. 1997, A\&A, 318, 215

Schmitt, J. H. M. M., \& Robrade, J. 2007, A\&A, 462, L41

Schmitt, J. H. M. M., Robrade, J., Ness, J.-U., Favata, F., \& Stelzer, B. 2005, A\&A, 432, L35

Smith, R. K., Brickhouse, N. S., Liedahl, D. A., \& Raymond, J. C. 2001, ApJ, 556, L91

Stelzer, B., \& Schmitt, J. H. M. M. 2004, A\&A, 418, 687

Telleschi, A., Güdel, M., Briggs, K. R., Audard, M., \& Scelsi, L. 2007, A\&A, 468,443

Vrba, F. J., Chugainov, P. F., Weaver, W. B., \& Stauffer, J. S. 1993, AJ, 106, 1608

Walter, F. M., \& Kuhi, L. V. 1981, ApJ, 250, 254

White, R. J., \& Basri, G. 2003, ApJ, 582, 1109

White, R. J., \& Hillenbrand, L. A. 2004, ApJ, 616, 998 